\title{
UHRF1 gene silencing inhibits cell proliferation and promotes cell apoptosis in human cervical squamous cell carcinoma CaSki cells
}

\author{
Ting-Ting Ge, Meng Yang, Zhuo Chen, Ge Lou* and Tao Gu
}

\begin{abstract}
Background: Up-regulation of UHRF1 has been observed in a variety of cancers and appears to serve as an independent prognostic factor.

Objective: To explore the effect of UHRF1 gene silencing on apoptosis and proliferation of cervical squamous cell carcinoma (CSCC) CaSki cells.

Methods: This study consisted of 47 CSCC tissues and 40 normal cervical tissues. The CaSki cells were assigned into Blank group (CaSki cells not transfected), NC group (CaSki cells transfected with control siRNA), and UHRF1 Silence group (CaSki cells transfected with UHRF1 siRNA). qRT-PCR and Western blot were used for UHRF1 mRNA and protein expressions, CKK-8 assay for cell proliferation, flow cytometry for cell cycle and apoptosis, Western blot for expressions of apoptosis-related proteins. Nude mice tumor transplant experiment was performed.

Results: UHRF1 exhibited higher mRNA and protein expressions in the CSCC tissues than normal cervical tissues (both $P<0.05$ ). The cell proliferation ability in the UHRF1 Silence group was reduced when compared with the Blank group and the NC group, the cells at S-G2M stage in the UHRF1 Silence group were dropped when compared with the Blank group and the NC group $(P<0.05)$, while the cells at $\mathrm{G} 0 / \mathrm{G} 1$ stage were elevated $(P<0.05)$, and the proportion of Annexin $V$ positive cells in the UHRF1 Silence group was increased in comparison with the Blank group and the NC group $(P<0.05)$. Nude mice tumor transplant experiment indicated that the growth rate and weight of tumor in the Blank group and NC group was higher and heavier than the UHRF1 Silence group $(P<0.05)$.

Conclusion: UHRF1 showed a high expression in CSCC and UHRF1 silencing can reduce proliferation and enhance apoptosis of the CaSki cells.
\end{abstract}

Keywords: UHRF1, Cervical squamous cell carcinoma, CaSki cell line, Proliferation, Apoptosis

\section{Background}

Cervical cancer is the third most commonly diagnosed gynecologic cancer and the fourth major cause of deathrelated cancer in females worldwide, accounting for $9 \%$ of the total new diagnoses and $8 \%$ of the total cancerassociated deaths among females in 2008 [1]. In China, there were 87,982 new patients diagnosed as cervical cancer and 23,375 deaths in 2011, and this disease has become one of the heaviest health burdens among Chinese women [2]. The major cause of cervical cancer

\footnotetext{
* Correspondence: hilouge@126.com

Department of Gynecology, Harbin Medical University Cancer Hospital, No. 150 Haping Road, Nangang District, Harbin 150040, Heilongjiang Province, People's Republic of China
}

refers to persistent human papillomavirus (HPV) infection, which is detected in $99 \%$ of patients with cervical tumors [3]. Interestingly, cervical cancer develops in a multi-step process that implicates the transformation of normal cervical epithelium into preneoplastic cervical intraepithelial neoplasia (CIN) that ultimately progress to invasive cervical cancer cells [4]. Accounting for $70 \sim 80 \%$ of cervical cancers, cervical squamous cell carcinoma (CSCC) consists of cells which are recognizably squamous but different from each other in term of growth pattern or cytological morphology [3]. The latest study reported there is no apparent symptom of early cervical cancer, while locally advanced disease may result in abnormal vaginal bleeding, pelvic pain, 
and dyspareunia [3]. Clearly, an improved understanding of the gene or molecular mechanisms via which pre-invasive lesions have the capability of invading the cervical stroma and eventually metastasis would exert significantly clinical influence.

UHRF1 [ubiquitin-like, containing plant homeodomain (PHD) and really interesting new gene (RING) finger domains 1], also known as ICBP90 or Np95, is a member of UHRF family and encode a $95-\mathrm{kDa}$ nuclear protein of 793 amino acids, with a single open reading frame (ORF) [5]. It contains an $\mathrm{N}$-terminal ubiquitylation-like domain, PHD, a SRA (SET and RING-associated) domain and a RING finger motif domain [6]. During the recent years, UHRF1 has received great concerns as a novel diagnostic marker of cancer [7]. UHRF1 is featured by a SRA domain (Set and Ring Associated) only found in the UHRF family, and up-regulation of UHRF1 has been observed in a variety of cancers, such as lung cancer and bladder cancer $[8,9]$. Also, previous studies demonstrated that the expression level of UHRF1 is closely associated with clinical stage, metastatic and prognostic of bladder cancer and lung cancer [7, 9]. Furthermore, over-expression of UHRF1 is shown to contribute to worse survival of laryngeal squamous cell carcinoma (LSCC) and serve as an independent prognostic factor of LSCC [10]. Therefore, the present study aims at exploring the effects of silent UHRF1 on proliferation and apoptosis of human CSCC Caski cells and to further study the related mechanism of cell apoptosis.

\section{Methods}

\section{Tissue collection and cell culture}

A total of 47 cases of CSCC tissues and 40 cases of normal cervical tissues resected from benign tumors were collected from Harbin Medical University Cancer Hospital from June 2013 to December 2015. The normal tissues were classified as the Control group and the CSCC tissues were as the CSCC group. The CSCC cell lines CaSki were obtained from cell bank of Chinese Academy of Sciences. The cells were cultured in 90 \% RPMI1640 (Gibco BRL Co. Led., New York, USA) and $10 \%$ Fetal bovine serum (FBS) (Gibco BRL Life Technologies Inc., Grand Island, New York, USA). UHRF1 siRNA and control siRNA were purchased from Santa Cruz (sc-76805, Santa Cruz Biotechnology Inc., Santa Cruz, CA, USA). When the cells merged to $50 \%$, lentivirus diluted by medium solution were added for transfection. After $24 \mathrm{~h}$, the virus was aspirated and normal solution was replaced. The cells were classified into 3 groups: the Blank group (CaSki cells untransfected), the negative control (NC) group (CaSki cells transfected with control siRNA) and the UHRF1 Silence group (CaSki cells transfected with UHRF1 siRNA).

\section{Quantificational real-time polymerase chain reaction (qRT-PCR)}

qRT-PCR was used for the detection of UHRF1 expression level in 47 cases of CSCC tissues, 40 normal cervical tissues and differenttransfected CaSki cells (Blank group, NC group, and UHRF1 Silence group). The tissues were washed 3 times with phosphate buffered saline (PBS). After 72-h culture, the cells were washed again with PBS for 3 times. The total RNA extraction was according to RNAiso Plus reagent kit (Takara Bio Inc., Dalian, China) and reverse transcription was operated in conformity with PrimeScript RT reagent kit (Takara Bio Inc., Dalian, China). The SteponePlus PCR (Applied Biosystem, Foster City, CA, USA) system applied for fluorescence quantitative test was following: cDNA solution 1.6 ul, 2X SYBR GREEN Taq PCR MIX (Takara Bio Inc., Dalian, China) 5 ul, PCR upstream and downstream primers $(10 \mathrm{uM}$, each $0.2 \mathrm{ul})$ and deuterium depleted water (DDW) 3 ul. Reaction condition was initial denaturation at $95{ }^{\circ} \mathrm{C}$ for $5 \mathrm{~min}, 95{ }^{\circ} \mathrm{C} 10 \mathrm{~s}, 58{ }^{\circ} \mathrm{C}$ $10 \mathrm{~s}$, and $72{ }^{\circ} \mathrm{C}$ for $10 \mathrm{~s}, 60$ cycles and extension at $72{ }^{\circ} \mathrm{C}$ for $10 \mathrm{~min}$. The detection gene was UHRF1 (Gene ID 29128) and internal reference gene was $\beta$-actin (Gene ID 11461). The primers (Invitrogen Biotechnology Co. Shanghai, China) were listed in Table 1 . The experiment was repeated 3 times, and the average values were obtained.

\section{Western blot assay}

After transfection of 3 days, the cells were washed, added with Radio immunoprecipitation assay (RIPA) lysis buffer and protease inhibitor (Sigma, St. Louis, USA) for homogenate, and centrifuged at $12,000 \times \mathrm{g}$ at $4{ }^{\circ} \mathrm{C}$ for $10 \mathrm{~min}$. After bicinchoninic acid (BCA) (Boi-rad Laboratories, Inc, Hercules, California, USA) detection of the protein concentration, the supernatant was reserved at $-80{ }^{\circ} \mathrm{C}$. Western blot applied $10 \%$ sodium dodecyl sulfate polyacrylamide gel electropheresis (SDS-PAGE) gel. Each well was added with 20 ug protein samples. The primary anti-bodies were Rabbit anti-human-UHRF1, Caspase3, Caspase8, Caspase9, Bax and Bcl-2 (Santa Cruz Biotechnology Inc., Santa Cruz, CA, USA) and the cells were kept overnight at $4{ }^{\circ} \mathrm{C}$. The secondary anti-body was POD-conjugated goat anti-rabbit (1:5000) and the cells were maintained at room temperature for $30 \mathrm{~min}$, which was added with horseradish peroxidase (HRP) (Boi-rad

Table 1 PCR primer sequences

\begin{tabular}{ll}
\hline Gene & Primer sequence \\
\hline UHRF1 & Forward: 5'- ACCAAGGTGGAGCCCTACAG -3' \\
& Reverse: 5'- CACTTAACTCAGGAACAACTGGAAC -3' \\
B-actin & Forward: 5'-CATCACGTACCAAACTTCAA-3' \\
& Reverse: 5'-CATCACAGTACCGGATTGC-3' \\
\hline
\end{tabular}


Laboratories, Inc, Hercules, California, USA). Image Quant 350 and Image Quant TL-1 (GE Healthcare, Fairfield, CT, USA) were applied to analyze the results. The reference gene was $\beta$-actin. The experiment was repeated 3 times, and the average values were obtained.

\section{CCK-8 for cell proliferation}

The CaSki cells were cultured in 96-hole plate at density of $1 \times 10^{5}$ cells. Each group was designed with 4 duplicated wells. After 24-h culture, the cell proliferation was examined with Cell Counting Kit-8 (Thermo, USA) at 24, 48, 72 and $96 \mathrm{~h}$. The procedures were as follows: After the aspiration of the original medium, the cells were added with CCK- 8 solution. The medium was detected at $450 \mathrm{~nm}$ absorbance after 2 -h culture. The experiment was repeated 3 times, and the average values were obtained.

\section{Flow cytometer}

Propidium iodide (PI) was applied to analyze cell cycle, and Annexin V-FITC and PI (Abcam Co., Cambridge, MA, USA) were used for analysis of cell apoptosis rate. The CaSki cells were washed twice with PBS and added with $0.25 \%$ pancreatin for digestion. After adjusting the density to $10^{5} / \mathrm{ml}$, the cells were incubated with fluorescent antibody at room temperature for $30 \mathrm{~min}$. The cells were precipitated and suspended after $200 \times \mathrm{g}$ centrifuging. Finally, the flow cytometer (BD Bioscience, San Jose, CA, USA) was applied for analysis. The experiment was repeated 3 times, and the average values were obtained.

\section{Establishment of tumor-bearing nude mice model}

A total of 24 female nude mice of 4-6 weeks were (purchased from Shanghai Biomodel Organism Science \& Technology Development Co., Ltd.) fed under sun protection factor (SPF) environment. The nude mice were injected with the CaSki cells in aseptic condition, and then were assigned into 3 groups (each group with 8 mice): the Blank group (injected with untransfected CaSki cell), the NC group (injected with CaSki cells transfecting control siRNA) and the UHRF1 Silence group (injected with CaSki cells transfecting UHRF1 siRNA). The cell number injected was $1 \times 10^{6}$ and the volume was $100 \mathrm{ul}$. Ten days later, the maximum and minimum diameter of the tumor was measured with vernier caliper every 5 days. The tumor size $=$ maximum diameter $\times$ minimum diameter ${ }^{2}$. Thirty days later, the tumor tissue on the back of nude mice were taken out and measured the weight. The tissues were fixed with $10 \%$ formaldehyde. After dehydration, the tissues were embedded with paraffin for staining analysis.

\section{Immunohistochemical staining}

The tissues were washed with PBS for 3 times and fixed with $4 \%$ paraformaldehyde. After embedding with paraffin and slicing, the tissues were performed immunohistochemical analysis. Firstly, the paraffin sections were dewaxed and rehydrated and treated with $3 \%$ hydrogen peroxide for 15 min to block endogenous peroxidase activity. Then the tissues were heated with vapor for $30 \mathrm{~min}$ to restore antigen. The tissues were later blocked in PBS chamber containing $1 \%$ bovine serum albumin (BSA). The primary antibody was added for incubation at room temperature for $30 \mathrm{~min}$ and then maintained overnight at $4{ }^{\circ} \mathrm{C}$. The primary antibody was rabbit polyclonal antiproliferating cell nuclear antigen (PCNA) and UHRF1 (Abcam Co., Cambridge, MA, USA) and the slicess were kept overnight at $4{ }^{\circ} \mathrm{C}$. The secondary antibody was PODconjugated goat anti-rabbit $(1: 5000)$ and the slices were maintained at room temperature for $30 \mathrm{~min}$, which was added with horseradish peroxidase (HRP) (Boi-rad Laboratories, Inc, Hercules, California, USA). The stained slices were pictured at $400 \times$ amplification.

\section{Statistical methods}

Data analysis was based upon the statistical package for the social sciences (SPSS) version 20.0 (SPSS Inc.; Chicago, IL, USA). Continuous data were displayed as mean \pm standard deviation, and differences between two groups were examined by $t$ test. And the differences among multiple groups were analyzed with repeated measurement of analysis of variance (ANOVA). Significance was illustrated by $P<0.05$.

\section{Results}

\section{UHRF1 expression by qRT-PCR}

The fluorescent quantitative PCR indicated that the mRNA level of UHRF1 was elevated in the tissues of the CSCC group in comparison with that in the Control group $(P<0.05)$. And the UHRF1 mRNA expression in the UHRF1 Silence group was lower than those in the Blank group and the NC group $(P<0.05)$. No significant difference was found regarding UHRF1 mRNA level between the Blank group and the NC group $(P>0.05$, Fig. 1). The findings confirmed that UHRF1 transcription level was promoted in the CSCC tissues when compared with the normal tissues and it could be inhibited by UHRF1 siRNA in the CaSkin cell lines.

\section{UHRF1 expression by Western blot assay}

The results of Western blot revealed that UHRF1 protein expression in the CSCC group was higher than that in the Control group $(P<0.05)$. And the UHRF1 protein level in the UHRF1 Silence group was reduced when compared with the Blank group and the NC group $(P<0.05)$. No significant difference was found regarding UHRF1 protein level between the Blank group and the NC group $(P>0.05$, Fig. 2$)$. It was verified that UHRF1 expression was increased in 

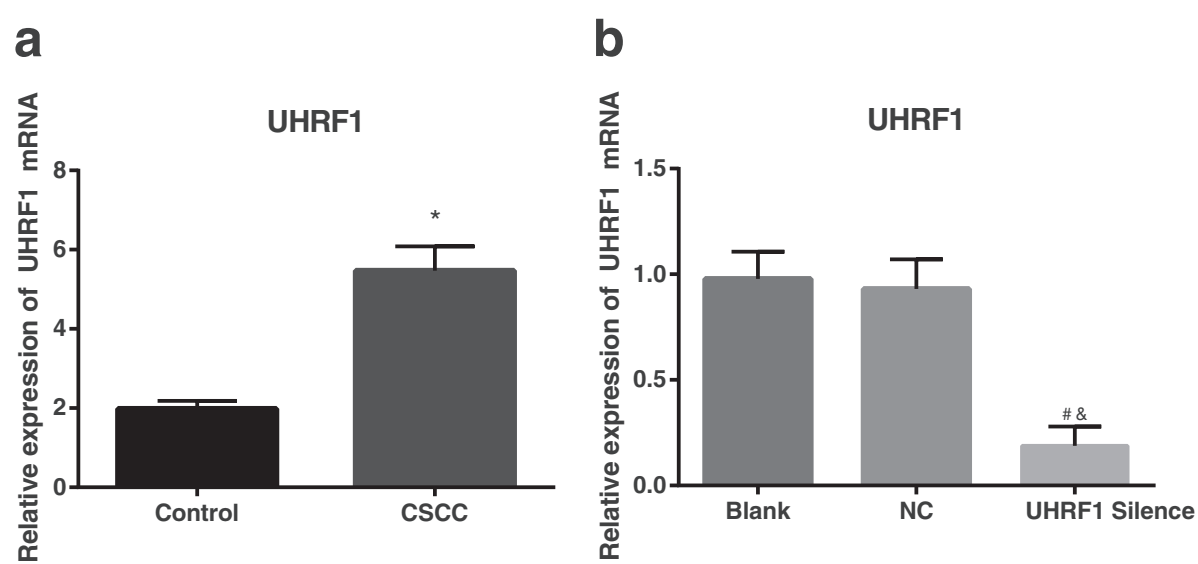

Fig. 1 UHRF1 mRNA expression detected by quantificational real-time polymerase chain reaction. a Comparison in UHRF1 expression between CSCC tissues $(n=47)$ and normal cervical tissues $(n=40)$, revealing that UHRF1 expression in CSCC tissues was much higher than that in normal cervical tissues; ${ }^{*}, P<0.05$ compared with the Control group; $\mathbf{b}$ Comparison in UHRF1 expression among CaSki cells of different transfection groups $(n=3)$, revealing that the UHRF1 Silence group had a significantly lower level of UHRF1 mRNA expression than the Blank group and the NC group; \#, $P<0.05$ compared with the Blank group; $\&, P<0.05$ compared with the NC group; UHRF1, ubiquitin-like, containing plant homeodomain (PHD) and really interesting new gene (RING) finger domains 1; CSCC, cervical squamous cell carcinoma; NC, negative control
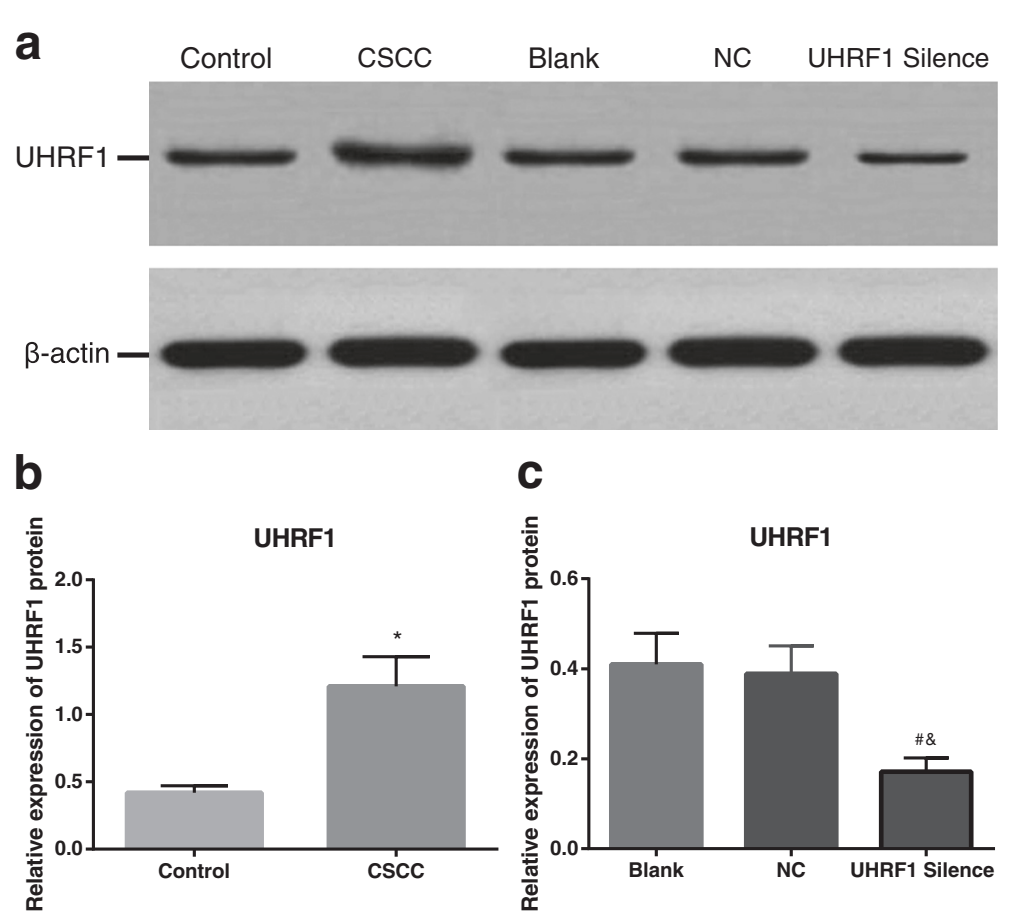

Fig. 2 UHRF1 protein expression detected by Western blot. a Western blot map of CSCC tissues $(n=47)$, normal cervical tissues ( $n=40)$ and CaSki cells in different transfection groups $(n=3)$; b Comparison of UHRF1 protein expression between CSCC tissues $(n=47)$ and normal cervical tissues $(n=40)$, showing that CSCC tissues had a significantly higher UHRF1 protein expression level than normal cervical tissues; ${ }^{*}, P<0.05$ compared with the Control group; c Comparison of UHRF1 protein expression among CaSki cells in different transfection groups $(n=3)$, showing that the UHRF1 Silence group had a significantly lower level of UHRF1 protein expression than the Blank group and the NC group; \#, $P<0.05$ compared with the Blank group; \& $P<0.05$ compared with the NC group; UHRF1, ubiquitin-like, containing plant homeodomain (PHD) and really interesting new gene (RING) finger domains 1 ; CSCC, cervical squamous cell carcinoma; NC, negative control 
the CSCC tissues when compared with the normal tissues and it could be inhibited by UHRF1 siRNA in the CaSkin cell lines.

\section{Cell proliferation by CCK-8}

It was found that the cell proliferation ability in the UHRF1 Silence group was reduced when compared with the Blank group and the NC group (both $P<0.05$ ). And there was no significant difference between the Blank group and the NC group $(P>0.05$, Fig. 3$)$. It was suggested that the proliferation ability of the CaSki cells was suppressed by UHRF1 siRNA.

\section{Cell cycle by flow cytometer}

The cells at S-G2M stage in the UHRF1 Silence group were dropped when compared with the Blank group and the NC group $(P<0.05$, Fig. 4$)$, while the cells at G0/G1 stage were elevated $(P<0.05)$. The UHRF1 Silence group induced the apoptosis of CaSki cells, which could be substantiated by the appearance of sub-G1 peak in the flow cytometric DNA histogram. The peak appeared ahead of the G0/G1 peak, and represented cell apoptosis. No significant difference existed between the Blank group and NC group $(P>0.05)$. Therefore, inhibiting U HRF1 was able to repress cell proliferation to stagnate the CSCC cells at G0/G1 stage.

\section{Cell apoptosis by flow cytometer}

It was found that the proportion of Annexin $\mathrm{V}$ positive cells (early apoptosis cells and late apoptosis cells) in the UHRF1 Silence group was increased in comparison with the Blank group and the NC group $(P<0.05$, Fig. 5$)$. No significant difference was found between the Blank group and the NC group $(P>0.05)$. Thus, the cell apoptosis rate was elevated when UHRF1 was inhibited.

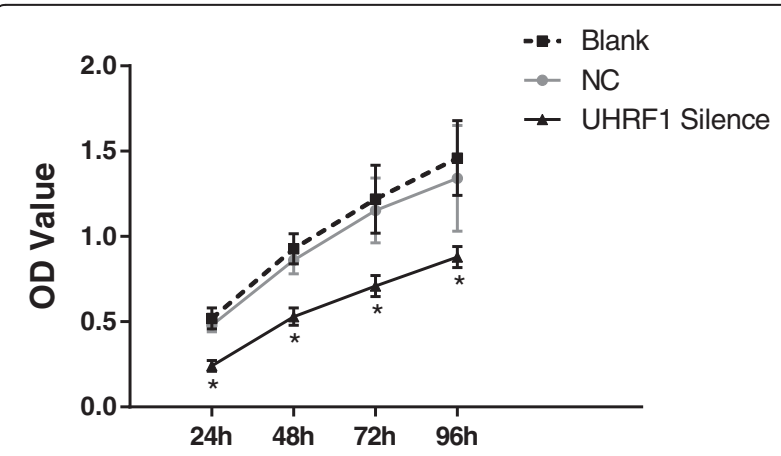

Fig. 3 Cell proliferation detected by CCK-8. At each time point, the UHRF1 Silence group had a much lower proliferation ability than the Blank group and the NC group; ${ }^{*}, P<0.05$ compared with the Control group, $n=3$; UHRF1, ubiquitin-like, containing plant homeodomain (PHD) and really interesting new gene (RING) finger domains 1; $N C$, negative control

\section{Apoptosis protein expressions by Western blot}

The expressions of the apoptosis proteins Caspase3, Caspase8, Caspase9 and Bax and anti-apoptosis protein $\mathrm{Bcl}-2$ in the Blank group were not significantly different from those in the NC group (all $P>0.05$ ). However, the expressions of above-mentioned apoptosis proteins in the UHRF1 Silence were elevated, while the antiapoptosis protein was reduced (all $P<0.05$, Fig. 6), which further confirmed that the inhibition of UHRF1 promoted cell apoptosis of the CaSki cells.

\section{Cell malignant transformation in tumor-bearing nude mice model}

The experiment indicated that the tumor growth speed in the UHRF1 Silence group was slower than that in the Blank group and NC group $(P<0.05$, Fig. $7 \mathrm{a})$. The tumor in the UHRF1 Silence group was also thinner than that in the Blank group and the NC group $(P<0.05$, Fig. $7 b)$. The slice staining results suggested that the UHRF1 and PCNA positive cells in the UHRF1 Silence group were less than the Blank group and the NC group (Fig. 7c), which further indicated that in the tumor-bearing nude mice model, the cell proliferation in the UHRF1 Silence group were significantly lower than the Blank group and the NC group. Therefore, the silent UHRF1 could inhibit the cell malignant transformation in the CaSki cells of the nude mice.

\section{Discussion}

As a leading cause of mortality in women, cervical cancer still exhibits unclear molecular mechanisms [4]. Despite of the significant advances including surgical techniques, radiotherapy and chemotherapy, there are still nearly $30 \%$ of patients diagnosed with invasive cervical carcinoma that passes away due to residual or recurrent disease [11]. However, increasing evidence indicates that the expression or overexpression of UHRF1 is associated with a poor prognosis in a variety of cancer types [7,9]. Therefore, the aim of the current study was to evaluate the potential association between UHRF1 and CSCC. Consequently, UHRF1 is over-expressed in CSCC and silencing UHRF1 inhibits proliferation and promotes apopyosis of the CSCC CaSKi cells.

In this study, we firstly assessed the expression level of UHRF1 in the CSCC tissues. We found that UHRF1 mRNA and protein were highly regulated in CSCC tissues in comparison to the normal cervical tissue, suggesting that UHRF1 participates in the development and evolvement of CSCC. Similarly, Yan et al. supported that UHRF1 has been considered to be an oncogene which is involved in tumorigenesis in ovarian cancer [6]. To our knowledge, UHRF1 can form a complex with DNA methyltransferase 1 (DNMT1) as well as histone 

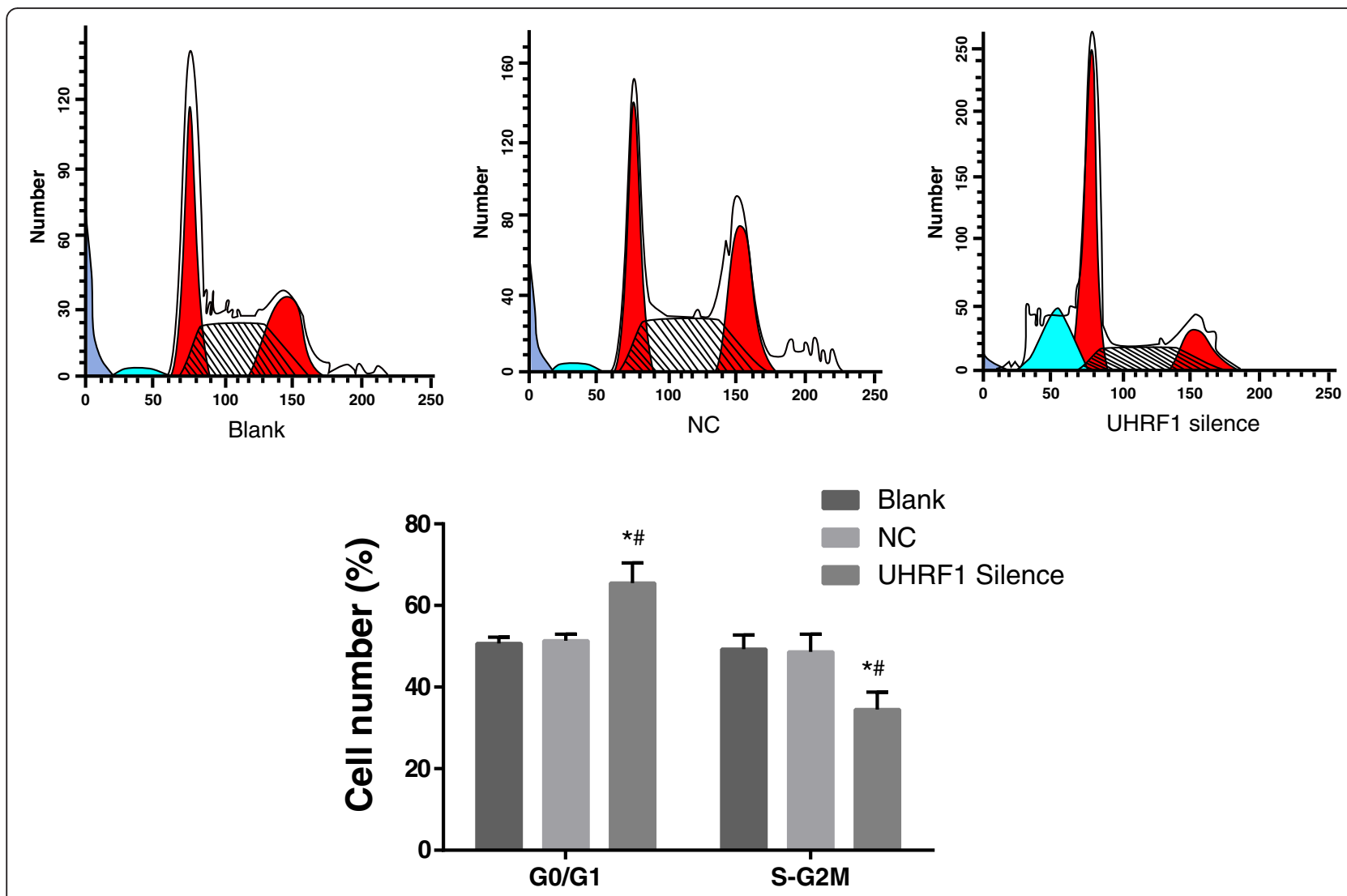

Fig. 4 Cell cycle detected by flow cytometer. Compared with the Blank group and the NC group, the ratio of S-G2M phase cells in UHRF1 Silence group decreased significantly, while the ratio of G0/G1 phase cells increased obviously. The UHRF1 Silence group induced the apoptosis of CaSki cells, which could be substantiated by the appearance of sub-G1 peak in flow cytometric DNA histogram. The peak appeared ahead of the G0/ G1 peak, and represented cell apoptosis. ${ }^{*}, P<0.05$ compared with the Blank group; \#, $P<0.05$ compared with the NC group; $n=3 ;$ UHRF1, ubiquitin-like, containing plant homeodomain (PHD) and really interesting new gene (RING) finger domains 1; NC, negative control

deacetylase 1 (HDAC1) and can restrict the expression of some tumor suppressor genes through its SRA domain, such as p16 INK4A, hMLH1, BRCA1 and RB1 [8]. According to former studies, high expression of UHRF1 was also detected in lung cancer cells, particularly in prostate cancer and breast cancer and it seem that the intensity of its over-expression was linked to the clinical stage of the cancer $[12,13]$. UHRF1 has recently been shown to play an important role in DNA methylation maintenance [14]. It has the ability to trigger the collection of DNMT and histone deacetylace- 1 by binding hemimethylated DNA via its SET- and RING-associated domain [15-17]. Krifa et al. and Alhosin et al. have proved that UHRF1, as an oncogene protein, has the potential to suppress tumor suppressor gene expression such as p16INK4A, hMLH1, BRCA1 along with RB1 through binding to methylated DNA and recruiting the DNMT1 $[8,18]$. Also, several studies indicate that overexpression of UHRF1 plays a part in the changed DNA methylation patterns and the establishment of aberrant histone code $[19,20]$.
Importantly, UHRF1 is essential for cell proliferation, and depleting UHRF1 may inhibit cell proliferation and lead to cell death. The results indicated that silencing UHRF1 leads to a decrease in the proliferation of CaSki cells and the apoptosis of CaSKi increases (the activity of Caspase3, Caspase8, Caspase9, Bax apoptosis-related protein increase). This is consistent with previous studies showing that down-regulation of UHRF1 leads to cell growth inhibition $[19,20]$. According to the research of $\mathrm{Li}$ et al., up-regulated UHRF1 might increase cyclin D1 activityand Bax downregulation, resulting in G1 shortage and inhibition of apoptosis and subsequent promotion of cell proliferation respectively [5]. As it was reported, the UHRF1 gene, its expression is associated with increased cell proliferation, is a target for the transcriptional activator E2F1 [21]. The results of nude mouse experiment indicated that tumor grow more slowly in UHRF1 silence group, implying that silencing UHRF1 can suppress the tumorigenesis of CaSKi cells in nude mouse. Moreover, UHRF1-knockout mouse embryonic stem cells exhibit significant loss of genomic methylation [14]. 


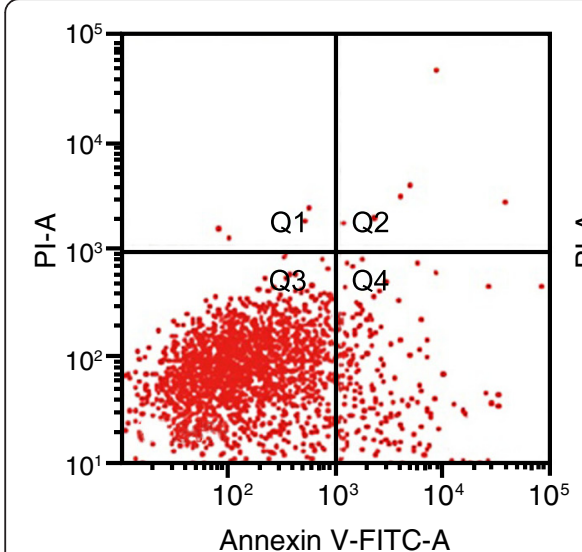

Blank

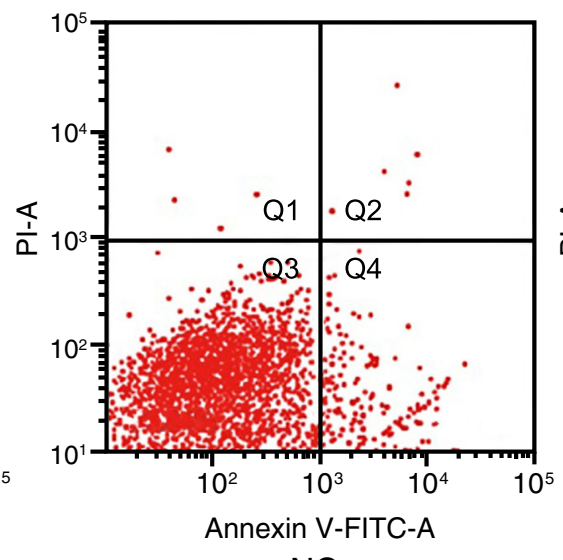

NC
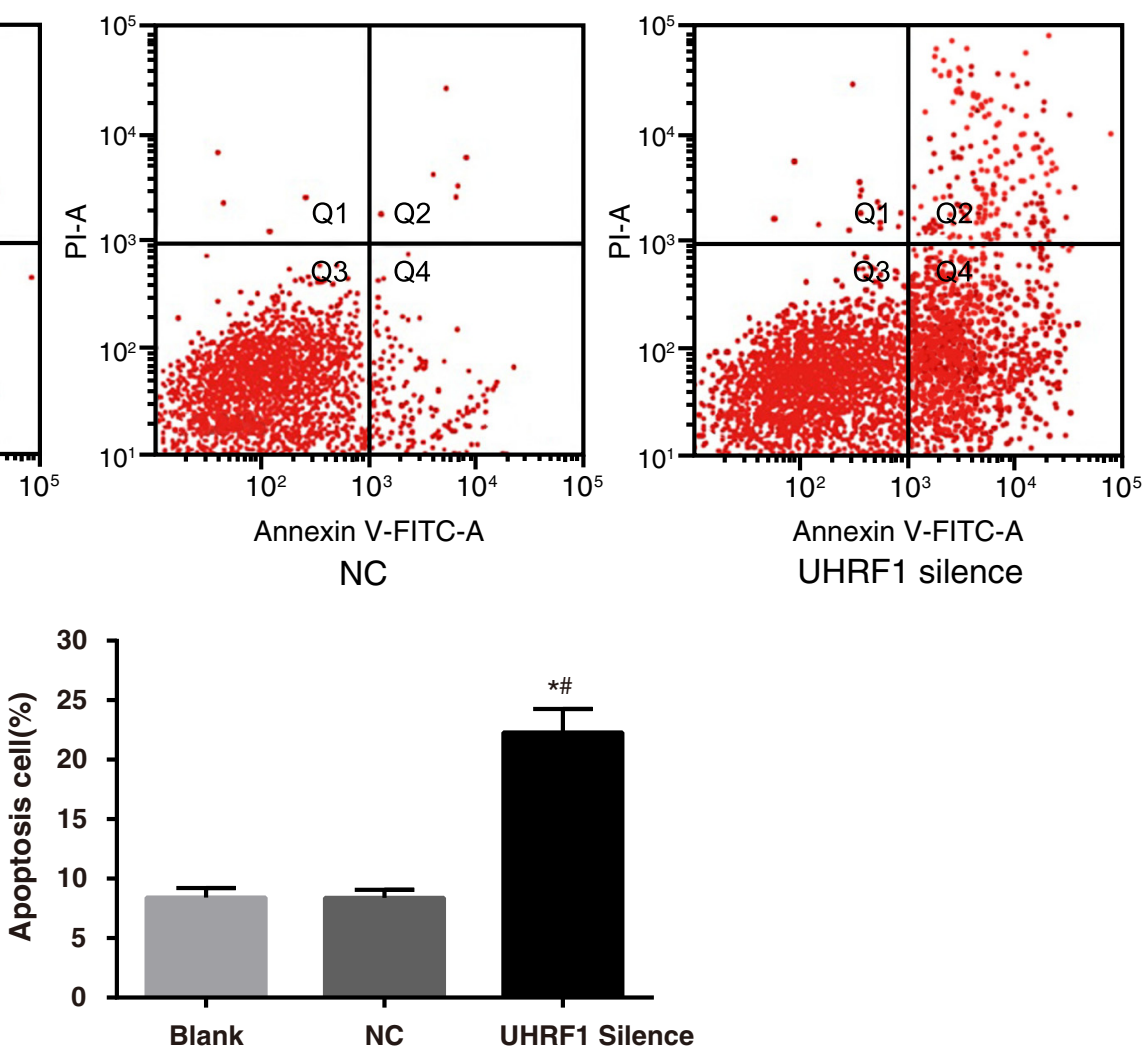

Fig. 5 Cell apoptosis detected by flow cytometer. Annexin V + PI - represents early apoptosis cells (Q4), annexin V + PI + represents late apoptosis cells (Q2). The Annexin V-positive cells (early and late apoptosis cells) in the UHRF1 Silence group increased evidently as compared with the Blank group and the NC group, which indicated increased apoptosis cells. *, $P<0.05$ compared with the Blank group; \#, $P<0.05$ compared with the NC group; $n=3$; UHRF1, ubiquitin-like, containing plant homeodomain (PHD) and really interesting new gene (RING) finger domains 1 ; NC, negative control

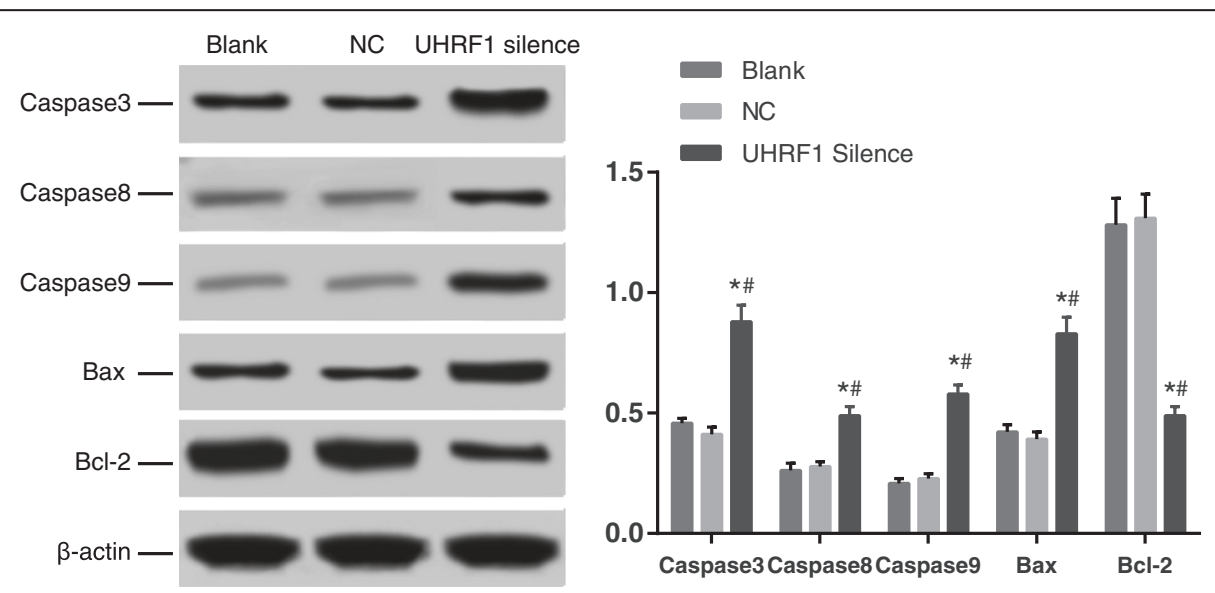

Fig. 6 Apoptosis related protein expression detected by Western blot. Compared with the Blank group and the NC group, apoptosis-related proteins, namely Caspase3, Caspase8, Caspase9 and Bax, had a much higher level in the UHRF1 Silence group, while anti-apoptotic protein ( $\mathrm{BCl}-2)$ had a much lower level. *, $P<0.05$ compared with the Blank group; $\#, P<0.05$ compared with the NC group, $n=3$; UHRF1, ubiquitin-like, containing plant homeodomain (PHD) and really interesting new gene (RING) finger domains 1 ; NC, negative control 


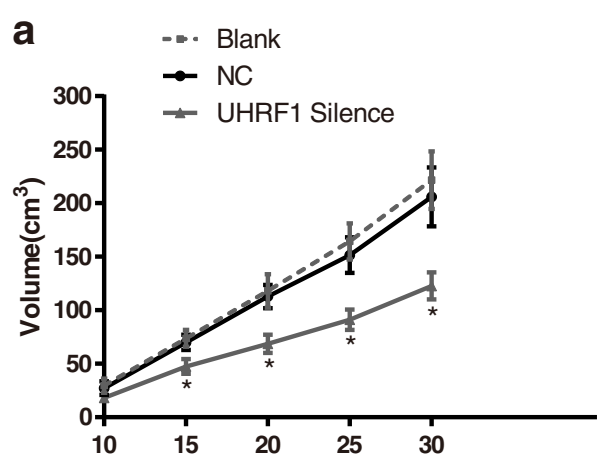

C

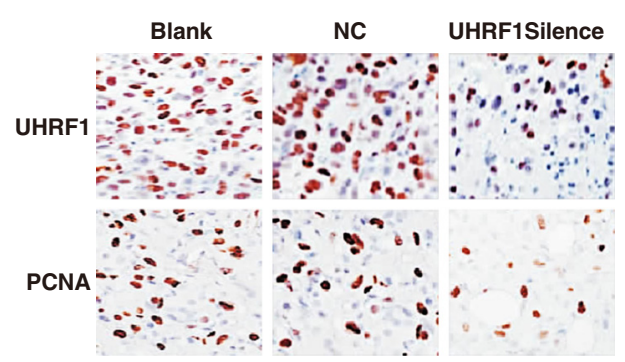

b

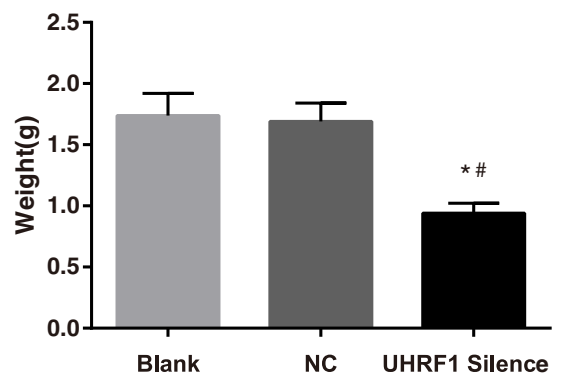

d

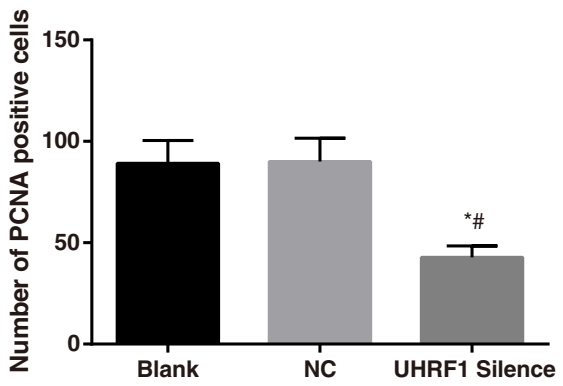

Fig. 7 Tumor-bearing nude mice model experiment. a growth curve of tumor volume. The tumor growth speed in the UHRF1 Silence group was much slower than that in the Blank group and NC group; $\mathbf{b}$ tumor weight. The tumor weight in the UHRF1 Silence group was much lower than that in the Blank group and the NC group; $\mathbf{c}$ immunohistochemical staining of tumor sections; $\mathbf{d}$ the number of PCNA-positive cells. The number of UHRF1-positive and PCNA-positive cells in the UHRF1 Silence group was much smaller than that in the Blank group and NC group. ${ }^{*}, P<0.05$ compared with the NC group; \#, $P<0.05$ compared with the NC group; UHRF1, ubiquitin-like, containing plant homeodomain (PHD) and really interesting new gene (RING) finger domains 1; NC, negative control; PCNA, proliferating cell nuclear antigen

Furthermore, in our study, the proliferation of CaSKi was prohibited when UHRF1 was down-regulating and cells are stay at G0/G1 stage. Alteration in UHRF1 expression is correlated with the degree of the lung cancer aggressiveness and it was detected in $50 \%$ of the patients in an early clinical stage [7]. Daskalos and his colleagues reported that UHRF1 is a prominent epigenetic switch that controls cell cycle in lung carcinoma due to its ability to smiantain the transcriptional silencing of tumor suppressor genes through sustaining their promoters in a hypermethylated condition [21]. UHRF1 mRNA and protein fluctuate with the cell cycle [22, 23], inactivation or loss of UHRF1 blocks S-phase entry [24], and zebrafish with a depletion-of-function mutation in UHRF1 have reduced cell proliferation and promoted cell apoptosis in hepatocyte [25]. These data suggests that up-regulating cancer cells of UHRF1 may result in cell death.

\section{Conclusions}

In conclusion, our study uncovered that UHRF1 overexpressed in CSCC and silent UHRF1 gene can reduce tumorigenicity of the CaSki cells by decreasing its proliferation capacity and making it stagnate at G0 and G1 phases as well as accelerating its apoptosis. Altogether, our data, combined with existing evidence provides a basis for investigating UHRF1 as a possible therapeutic target in human cancer.

\section{Abbreviations}

ANOVA, analysis of variance; BCA, bicinchoninic acid; BSA, bovine serum albumin; CIN, cervical intraepithelial neoplasia; CSCC, cervical squamous cell carcinoma; DDW, deuterium depleted water; DNMT1, DNA methyltransferase 1; FBS, fetal bovine serum; HDAC1, histone deacetylase 1; HPV, human papillomavirus; HRP, horseradish peroxidase; LSCC, laryngeal squamous cell carcinoma; NC, negative control; ORF, open reading frame; PBS, phosphate buffered saline; PCNA, proliferating cell nuclear antigen; PI, propidium iodide; qRT-PCR, quantificational real-time polymerase chain reaction; RIPA, radio immunoprecipitation assay; SDS-PAGE, sodium dodecyl sulfate polyacrylamide gel electropheresis; SPF, sun protection factor; SPSS, statistical package for the social sciences; SRA, Set and Ring-associated; UHRF1, ubiquitin-like, containing plant homeodomain and really interesting new gene finger domains 1

\section{Acknowledgements}

We would like to give our sincere gratitude to the reviewers for their comments.

\section{Authors' contributions}

TTG and MY carried out the molecular genetic studies, participated in the sequence alignment and drafted the manuscript. ZC carried out the immunoassays. GL participated in the sequence alignment. TG participated in the design of the study and performed the statistical analysis. FG conceived of the study and participated in its design and coordination. All authors read and approved the final manuscript. 


\section{Consent for publication}

Not applicable.

\section{Ethics approval and consent to participate}

All subjects have signed informed consents. This study was approved by Ethics Committee of Harbin Medical University Cancer Hospital.

Received: 14 May 2016 Accepted: 5 July 2016

Published online: 19 July 2016

\section{References}

1. Jemal A, Bray F, Center MM, Ferlay J, Ward E, Forman D. Global cancer statistics. CA Cancer J Clin. 2011;61:69-90.

2. $\quad$ Li Z, Yang Y, Gao Y, Wu X, Yang X, Zhu Y, Yang H, Wu L, Yang C, Song L. Elevated expression of flotillin-1 is associated with lymph node metastasis and poor prognosis in early-stage cervical cancer. Am J Cancer Res. 2016;6:38-50.

3. Colombo N, Carinelli S, Colombo A, Marini C, Rollo D, Sessa C, E. G. W. Group. Cervical cancer: ESMO Clinical Practice Guidelines for diagnosis, treatment and follow-up. Ann Oncol. 2012;23 Suppl 7:vii27-32.

4. Jiang $P$, Ren $Y L$, Li JL, Luo J. Girdin expression in cervical carcinoma and its role in the malignant properties of HeLa cells. Oncol Lett. 2016;11:2440-4.

5. $\mathrm{Li} \mathrm{XL}, \mathrm{Xu} \mathrm{JH}$, Nie JH, Fan SJ. Exogenous expression of UHRF1 promotes proliferation and metastasis of breast cancer cells. Oncol Rep. 2012;28:375-83.

6. Yan F, Wang X, Shao L, Ge M, Hu X. Analysis of UHRF1 expression in human ovarian cancer tissues and its regulation in cancer cell growth. Tumour Biol. 2015:36:8887-93.

7. Unoki M, Daigo Y, Koinuma J, Tsuchiya E, Hamamoto R, Nakamura Y. UHRF1 is a novel diagnostic marker of lung cancer. Br J Cancer. 2010;103:217-22.

8. Alhosin M, Sharif T, Mousli M, Etienne-Selloum N, Fuhrmann G, Schini-Kerth VB, et al. Down-regulation of UHRF1, associated with re-expression of tumor suppressor genes, is a common feature of natural compounds exhibiting anti-cancer properties. J Exp Clin Cancer Res. 2011;30:41.

9. Unoki M, Kelly JD, Neal DE, Ponder BA, Nakamura Y, Hamamoto R. UHRF1 is a novel molecular marker for diagnosis and the prognosis of bladder cancer. Br J Cancer. 2009;101:98-105.

10. Pi JT, Lin $Y$, Quan $Q$, Chen LL, Jiang LZ, Chi W, et al. Overexpression of UHRF1 is significantly associated with poor prognosis in laryngeal squamous cell carcinoma. Med Oncol. 2013;30:613.

11. Mahajan A, Engineer R, Chopra S, Mahanshetty U, Juvekar SL, Shrivastava SK et al. Role of 3 T multiparametric-MRI with BOLD hypoxia imaging for diagnosis and post therapy response evaluation of postoperative recurrent cervical cancers. Eur J Radiol Open. 2016;3:22-30.

12. Matsushita, R, Yoshino H, Enokida H, Goto Y, Miyamoto K, Yonemori M., Inoguchi S, Nakagawa M Sek Ni. Regulation of UHRF1 by dual-strand tumorsuppressor microRNA-145 (miR-145-5p and miR-145-3p): Inhibition of bladder cancer cell aggressiveness. Oncotarget. 2016; doi:10.18632/oncotarget.8668.

13. Wan X, Yang S, Huang W, Wu D, Chen H, Wu M, et al. UHRF1 overexpression is involved in cell proliferation and biochemical recurrence in prostate cancer after radical prostatectomy. J Exp Clin Cancer Res. 2016;35:34.

14. Bostick M, Kim JK, Esteve PO, Clark A, Pradhan S, Jacobsen SE. UHRF1 plays a role in maintaining DNA methylation in mammalian cells. Science. 2007;317:1760-4.

15. Arita K, Ariyoshi M, Tochio H, Nakamura Y, Shirakawa M. Recognition of hemi-methylated DNA by the SRA protein UHRF1 by a base-flipping mechanism. Nature. 2008;455:818-21.

16. Avvakumov GV, Walker JR, Xue S, Li Y, Duan S, Bronner C, Arrowsmith CH, Dhe-Paganon S. Structural basis for recognition of hemi-methylated DNA by the SRA domain of human UHRF1. Nature. 2008;455:822-5.

17. Sharif J, Muto M, Takebayashi S, Suetake I, Iwamatsu A, Endo TA, Shinga J, Mizutani-Koseki Y, Toyoda T, Okamura K, Tajima S, Mitsuya K, Okano M, Koseki $\mathrm{H}$. The SRA protein Np95 mediates epigenetic inheritance by recruiting Dnmt1 to methylated DNA. Nature. 2007:450:908-12.

18. Krifa M, Alhosin M, Muller CD, Gies JP, Chekir-Ghedira L, Ghedira K, Mely Y, Bronner C, Mousli M. Limoniastrum guyonianum aqueous gall extract induces apoptosis in human cervical cancer cells involving p16 INK4A reexpression related to UHRF1 and DNMT1 down-regulation. J Exp Clin Cancer Res. 2013:32:30.

19. Jenkins $Y$, Markovtsov V, Lang W, Sharma P, Pearsall D, Warner J, Franci C, Huang B, Huang J, Yam GC, Vistan JP, Pali E, Vialard J, Janicot $M$, Lorens JB, Payan DG, Hitoshi Y. Critical role of the ubiquitin ligase activity of UHRF1, a nuclear RING finger protein, in tumor cell growth. Mol Biol Cell. 2005;16:5621-9.
20. Arima Y, Hirota T, Bronner C, Mousli M, Fujiwara T, Niwa S, Ishikawa H, Saya H. Down-regulation of nuclear protein ICBP90 by p53/p21Cip1/WAF1dependent DNA-damage checkpoint signals contributes to cell cycle arrest at G1/S transition. Genes Cells. 2004:9:131-42.

21. Daskalos A, Oleksiewicz U, Filia A, Nikolaidis G, Xinarianos G, Gosney JR, Malliri A, Field JK, Liloglou T. UHRF1-mediated tumor suppressor gene inactivation in nonsmall cell lung cancer. Cancer. 2011;117:1027-37.

22. Sadler KC, Krahn KN, Gaur NA, Ukomadu C. Liver growth in the embryo and during liver regeneration in zebrafish requires the cell cycle regulator, uhrf1. Proc Natl Acad Sci U S A. 2007;104:1570-5.

23. Mousli $M$, Hopfner $R$, Abbady AQ, Monte $D$, Jeanblanc $M$, Oudet $P$, Louis $B$, Bronner C. ICBP90 belongs to a new family of proteins with an expression that is deregulated in cancer cells. Br J Cancer. 2003;89:120-7.

24. Bonapace IM, Latella L, Papait R, Nicassio F, Sacco A, Muto M, Crescenzi M, Di Fiore PP. Np95 is regulated by E1A during mitotic reactivation of terminally differentiated cells and is essential for $\mathbf{S}$ phase entry. J Cell Biol. 2002;157:909-14.

25. Tien AL, Senbanerjee S, Kulkarni A, Mudbhary R, Goudreau B, Ganesan S, Sadler KC, Ukomadu C. UHRF1 depletion causes a G2/M arrest, activation of DNA damage response and apoptosis. Biochem J. 2011;435:175-85.

\section{Submit your next manuscript to BioMed Central and we will help you at every step:}

- We accept pre-submission inquiries

- Our selector tool helps you to find the most relevant journal

- We provide round the clock customer support

- Convenient online submission

- Thorough peer review

- Inclusion in PubMed and all major indexing services

- Maximum visibility for your research

Submit your manuscript at www.biomedcentral.com/submit
) Biomed Central 